\title{
FIXED POINTS OF HOLOMORPHIC MAPS IN BANACH SPACES
}

\author{
T. L. HAYDEN ${ }^{1}$ AND T. J. SUFFRIDGE
}

\begin{abstract}
This paper is concerned with the problem of existence of fixed points of continuous maps of the closed unit ball of a complex Banach space into itself which are holomorphic on the open unit ball. We show that if the Banach space is separable and reflexive and $F$ is the map in question that for a.e. $\theta$ in $[0,2 \pi]$ the map $e^{i \theta} F$ has a fixed point. This result does not hold in general; hence, additional conditions are imposed which insure the existence of fixed points in every Banach space. Fixed points of some linear fractional maps are explicitly computed.
\end{abstract}

1. Introduction. Let $X$ be a complex Banach space, $U$ the open unit ball in $X, \bar{U}$ the closed unit ball and assume $F: \bar{U} \rightarrow \bar{U}$ is continuous on $\bar{U}$ and holomorphic on $U$. We denote the set of such functions by $\bar{U}_{H, C}$. By holomorphic we mean that $F$ has a complex linear Fréchet derivative at each point of $U$. Standard results on these maps and vector valued holomorphic maps may be found in the book of Hille and Phillips [8].

In a previous paper [6], the authors showed that a biholomorphic map of the unit ball of a Hilbert space into itself has a fixed point. An example of Kakutani exhibits a homeomorphism (which can easily be modified to a diffeomorphism) of the closed unit ball of a Hilbert space onto itself with no fixed point. This might indicate that holomorphy is strong enough to always insure fixed points. However we show in $\$ 2$ an example of a holomorphic map $F$ on the Banach space $c_{0}$ with no fixed point. In fact for no $\theta$ in $[0,2 \pi]$ does $e^{i \theta} F$ have a fixed point.

Earle and Hamilton [4] have shown that if $F$ is holomorphic on $U$ in a general Banach space and $F$ maps $U$ strictly inside $U$, then $F$ has a fixed point in $U$. Their results depend on the fact that one can define a metric on $U$ so that if $F$ maps $U$ into $U$ and is holomorphic, then $F$ is nonexpansive, and if $F$ maps $U$ strictly inside $U$ then $F$ is a strict contraction in this metric. This result is fundamental to our work and we have included some of their results in the appendix. The appendix is a generalization of some of their work and is due to L. Harris (unpublished).

$\$ 2$ contains the main result and is obtained by using a result of Ryan [15]

Received by the editors May 5, 1975.

AMS (MOS) subject classifications (1970). Primary 47H10.

Key words and phrases. Fixed points, holomorphic mappings, invariant metric.

1 This work was supported in part by the National Science Foundation under Grant GP-22577. 
on boundary values of vector valued holomorphic functions. We show that if $X$ is separable and reflexive and if $F: \bar{U} \rightarrow \bar{U}$ is continuous on $\bar{U}$ and holomorphic in $U$, then $e^{i \theta} F$ has a fixed point in $\bar{U}$ for a.e. $\theta$ in $[0,2 \pi]$. In $\S 3$, in addition to the assumption of holomorphy we impose several different conditions that are frequently assumed in fixed point theory for nonexpansive maps and show that these additional conditions are sufficient to obtain fixed points. In $\$ 4$ we compute explicitly the fixed points of certain linear fractional maps in the space $B(H, H)$ of bounded linear operators on a Hilbert space.

2. Fixed points of holomorphic maps in Banach spaces. We will show that for $|\alpha|<1$ the fixed points of the map $\alpha F$ are holomorphic functions of $\alpha$. Then we study the fixed points of $F$ by considering the boundary values of the vector valued holomorphic function $x(\alpha)$. Boundary values of such functions have been considered by Ryan [15]. We begin with

Lemma 2.1. Suppose $F: U \rightarrow U$ is holomorphic, and let $x(\alpha)=(\alpha F)(x(\alpha))$ be the unique fixed point of $\alpha F$ for $|\alpha|<1$. Then $x(\alpha)$ is a holomorphic function of $\alpha$.

Proof. By Theorem 2 of the appendix, $\alpha F$ is a strict contraction in the metric $\rho$ defined there, so $x(\alpha)=\lim _{n \rightarrow \infty}(\alpha F)^{n}(0)$. Let $x_{n}(\alpha)=(\alpha F)^{n}(0)$ and fix $r$ with $0<r<1$. We will show that $x_{n}(\alpha)$ converges uniformly to $x(\alpha)$ in the closed disc $|\alpha| \leqslant r$. Since $\alpha F$ maps $U$ into the ball of radius $r$, the results in the appendix imply that

$$
\rho\left((\alpha F)^{n}(u),(\alpha F)^{n}(v)\right) \leqslant k^{n} \rho(u, v)
$$

for all $u, v \in U$, where $k=2 /(3-r)<1$. Hence by the last result of the appendix,

$$
\begin{aligned}
\left\|x_{n}(\alpha)-x_{n+p}(\alpha)\right\| & \leqslant \rho\left((\alpha F)^{n}(0),(\alpha F)^{n}(\alpha F)^{p}(0)\right) \leqslant k^{n} \rho\left(0,(\alpha F)^{p}(0)\right) \\
& \leqslant k^{n} r /(1-r)
\end{aligned}
$$

for all $|\alpha| \leqslant r$, so the sequence of functions $\left\{x_{n}(\alpha)\right\}$ is uniformly Cauchy on $|\alpha| \leqslant r$. Also $\left\|x_{n}(\alpha)\right\| \leqslant r$ for $|\alpha|<r$. Hence for every $l \in X^{\prime}$ the dual of $X$, the sequence $\left\{l \circ x_{n}(\alpha)\right\}$ is a uniformly convergent sequence of analytic functions on $|\alpha| \leqslant r$ which converges to the function $l \circ x(\alpha)$, and hence $l \circ x(\alpha)$ is analytic. The equivalence of weak and strong holomorphy [8] implies that $x(\alpha)$ is holomorphic.

We review briefly the definitions and results of Ryan [15] needed for our paper. Let $\mathfrak{L}^{p}(T)$ for $1 \leqslant p<\infty$ denote the space of measurable complex valued functions defined on the unit circle $T$ and such that $N_{p}(f)$ $\equiv\left((2 \pi)^{-1} \int_{0}^{2 \pi}\left|f\left(e^{i \theta}\right)\right|^{p} d \theta\right)^{1 / p}<\infty$. $\mathcal{E}_{X}^{p}(T)$ denotes the space of functions defined a.e. on $T$ with values in $X$ which are measurable in the norm topology of $X$ and so that $\|f\| \in \mathcal{L}^{p}(T)$. If $f \in \mathcal{L}_{X}^{p}(T)$ denote $N_{p}(f)=N_{p}(\|f\|)$. For $x \in X$ and $y \in X^{\prime}$ the dual of $X$, denote by $\langle x, y\rangle$ the value of the linear functional $y$ at the point $x$. Since $X$ is separable, a function $f$ from $T$ to $X$ is measurable if and only if $\langle f, y\rangle$ is measurable for every $y \in X^{\prime}$. Let $\mathcal{E}_{X_{s}^{\prime}}^{p}$ denote 
the space of functions which are defined a.e. on $T$ with values on $X^{\prime}$, and so that $\langle x, f\rangle$ is measurable for each $x \in X$ and $\|f\| \in \mathfrak{L}^{p}(T)$. Again the separability of $X$ implies that $\|f\|$ is measurable if $\langle x, f\rangle$ is measurable for all $x \in X$. In general, $\mathcal{L}_{X^{\prime}}^{p}(T) \subset \mathcal{L}_{X_{s}^{\prime}}^{p}(T)$, but we have equality if $X$ is reflexive for then $X^{\prime}$ is separable. If $f \in \mathcal{E}_{X_{s}^{\prime}}^{p}$ then $\langle x, f\rangle \in \mathfrak{L}^{p}(T)$ for each $x \in X$ and the weak integral or Pettis integral of $f$ is a linear functional on $X$ defined by

$$
\left\langle x,(\mathrm{P}) \int_{0}^{2 \pi} f\left(e^{i \theta}\right) d \theta\right\rangle=\int_{0}^{2 \pi}\left\langle x, f\left(e^{i \theta}\right)\right\rangle d \theta .
$$

We will need the following two theorems of Ryan [15].

TheOREM A (RYAN). Let $X$ be separable and $f \in \mathcal{L}_{X^{\prime}}^{p}(T)$ for $1 \leqslant p<\infty$. If (P) $\int_{0}^{2 \pi} e^{i n \theta} f\left(e^{i \theta}\right) d \theta=0$ for $n=1,2,3, \ldots$, then $N_{p}\left(F_{r}\right) \leqslant N_{p}(f)$ for $0<r$ $<1$ where

$$
F(z)=\frac{1}{2 \pi i}(\mathrm{P}) \int_{|t|=1} \frac{f(t)}{t-z} d t .
$$

Furthermore $N_{p}\left(F_{r}-f\right) \rightarrow 0$ as $r \rightarrow 1$ and $F_{r}\left(e^{i \theta}\right) \rightarrow f\left(e^{i \theta}\right)$ a.e. in the norm of $X^{\prime}$. If $f$ is continuous then $F_{r}\left(e^{i \theta}\right) \rightarrow f\left(e^{i \theta}\right)$ uniformly for $\theta \in[0,2 \pi]$.

THEOREM B (RyAN). Suppose that $X$ is a separable Banach space with dual $X^{\prime}$. Let $F$ be analytic in $|z|<1$ with values in $X^{\prime}$ and assume $N_{p}\left(F_{r}\right) \leqslant 1$ for $0<r$ $<1$ where $1<p \leqslant \infty$ and $F_{r}\left(e^{i \theta}\right)=F\left(r e^{i \theta}\right)$. Then there is a function $f$ $\in \mathcal{L}_{X_{s}^{\prime}}^{p}(T)$ so that

$$
F(z)=\frac{1}{2 \pi i}(\mathrm{P}) \int_{|t|=1} \frac{f(t)}{t-z} d t
$$

and furthermore,

$$
N_{p}(f) \leqslant 1, \quad(\mathrm{P}) \int_{0}^{2 \pi} e^{i n \theta} f\left(e^{i \theta}\right) d \theta=0 \text { for } n=1,2,3, \ldots
$$

and $f$ is uniquely determined modulo a set of measure zero.

We now state our theorem on fixed points.

Theorem 2.2. Suppose $X$ is a separable reflexive Banach space, $U$ is the open unit ball of $X$ and $F: \bar{U} \rightarrow \bar{U}$ is continuous on $\bar{U}$ and holomorphic in $U$. Then for a.e. $\theta$ in $[0,2 \pi], e^{i \theta} F$ has a fixed point.

Proof. For $|\alpha|<1$ let $x(\alpha)=\alpha F(x(\alpha))$ be the fixed points of $\alpha F$. We note again that $x(\alpha)$ is a vector valued holomorphic function of $\alpha$.

Since $\left\|x\left(r e^{i \theta}\right)\right\| \leqslant r$, and $N_{p}\left(x_{r}\right)=\left((2 \pi)^{-1} \int_{0}^{2 \pi}\left\|x\left(r e^{i \theta}\right)\right\|^{p} d \theta\right)^{1 / p}$, we have $N_{p}\left(x_{r}\right) \leqslant 1$ for $0 \leqslant r<1$ and $p \geqslant 0$. Since $x(\alpha)$ has values in $X$, the dual of $X^{\prime}$, and since $X$ is reflexive, we have by Ryan's Theorem B that there is a function $y \in \mathcal{L}_{X^{\prime \prime}, s}^{p}(T)=\mathcal{L}_{X^{\prime \prime}}^{p}(T)=\mathcal{L}_{X}^{p}(T)$ so that 


$$
x(\alpha)=\frac{1}{2 \pi i}(\mathrm{P}) \int_{|t|=1} \frac{y(t)}{t-z} d t
$$

$N_{p}(y) \leqslant 1$ and $(\mathrm{P}) \int_{0}^{2 \pi} e^{i n \theta} y\left(e^{i \theta}\right) d \theta=0$ for $n=1,2,3, \ldots$

We may now use Ryan's Theorem A, and since $y \in \mathcal{L}_{X}^{p}$, we have that

$$
x(\alpha)=\frac{1}{2 \pi i}(\mathrm{P}) \int_{|t|=1} \frac{y(t)}{t-\alpha} d t
$$

and $x\left(r e^{i \theta}\right) \rightarrow y\left(e^{i \theta}\right)$ a.e. in the norm of $X$. The proof is finished by noting that the fixed points of $r e^{i \theta} F$ are $x\left(r e^{i \theta}\right)$ and that these have a limit as $r \rightarrow 1$ so that $y\left(e^{i \theta}\right)$ is the fixed point of $e^{i \theta} F$.

We now show that Theorem 2.2 fails in the nonreflexive space $c_{0}$. Let $X=c_{0}$, the space of complex sequences which tend to zero and with the norm in $c_{0}$ the supremum norm. Let $F: \bar{U} \rightarrow \bar{U}$ be defined by $F\left(z_{1}, z_{2}, z_{3}, \ldots\right)$ $=\left(\frac{1}{2}, z_{1}, z_{2}, z_{3}, \ldots\right)$. It is clear that $F$ is holomorphic in the entire space $X$ since $F$ is simply a translate of the linear shift operator. In fact $F$ is nonexpansive in the norm and it is well known that $F$ has no fixed points, since a fixed point must obviously be $\left(\frac{1}{2}, \frac{1}{2}, \frac{1}{2}, \ldots\right)$ which is not in $c_{0}$. In fact note that $e^{i \theta} F\left(z_{1}, z_{2}, \ldots\right)=\left(z_{1}, z_{2}, \ldots\right)$ implies $z_{1}=e^{i \theta} / 2, z_{2}=e^{2 i \theta} / 2, \ldots, z_{j}$ $=e^{i j \theta} / 2, \ldots ;$ a point not in $c_{0}$, so that for no $\theta$ in $[0,2 \pi]$ does $e^{i \theta} F$ have a fixed point.

3. Fixed points of holomorphic maps in general Banach spaces. The addition of a simple geometrical condition to holomorphy yields the following theorem.

TheOREM 3.1. Suppose $F \in \bar{U}_{H, C}$ and $(I-F) \bar{U}$ is closed; then $F$ has a fixed point in $\bar{U}$.

Proof. For $t \in(0,1)$, let $x(t)=t F(x(t))$ be the fixed points of the map $t F$. Then

$$
\|x(t)-F(x(t))\|=\|t F(x(t))-F(x(t))\| \leqslant|t-1|\|F(x(t))\| \leqslant|t-1| .
$$

Hence $x(t)-F(x(t)) \rightarrow 0$ as $t \rightarrow 1$. Hence $0 \in(I-F) \bar{U}$ so that $F$ has a fixed point.

Such arguments are standard for nonexpansive maps and we note the addition to the hypothesis that $F \in \bar{U}_{H, C}$ of a condition such as demicompact or demiclosed in the appropriate setting will yield fixed points as in the nonexpansive case. The notions of demicompact and demiclosed are used by Browder and Petryshyn, [2], [3], [13]. The reader can easily formulate and prove the corresponding results for holomorphic maps.

Theorem 3.2. Let $F \in \bar{U}_{H, C}$ and for $0 \leqslant t<1$, let $x(t)$ be the unique fixed point of $t F$. Let $D F_{x(t)}$ be the Fréchet derivative of $F$ at $x(t)$. If 1 is not in the spectrum of $D F_{x(t)}$ and $\left\|\left(I-t D F_{x(t)}\right)^{-1}\right\|=g(t)$ is integrable on $(0,1)$, then $F$ has a fixed point in $\bar{U}$.

Proof. By Lemma $2.1 x(t)$ is a differentiable function of $t$. Hence since 
$x(t)=t F(x(t))$ we have that

$$
\dot{x}(t)=F(x(t))+t D F_{x(t)} \dot{x}(t), \quad \text { or } \quad\left(I-t D F_{x(t)}\right) \dot{x}(t)=x(t) / t
$$

$$
\text { for } 0<t<1 \text {. }
$$

Since $\|x(t)\| \leqslant t$ it follows that $\|\dot{x}(t)\| \leqslant\left\|\left(I-t D F_{x(t)}\right)^{-1}\right\|=g(t)$. Since the length of the path of $x(t)$ is given by $\lim _{s \rightarrow 1} \int_{0}^{s}\|\dot{x}(t)\| d t \leqslant \lim _{s \rightarrow 1} \int_{0}^{s} g(t) d t$ $<\infty$, we see that there is some $x$ so that $x(t) \rightarrow x$ as $t \rightarrow 1$ and that $x=F(x)$.

Following Lumer and Phillips [10], we define a semi-inner-product on $X$. The semi-inner-product is a map $[\cdot, \cdot]$ from $X \times X \rightarrow C$ (the complex numbers) so that:

$$
\begin{aligned}
{[x+y, z] } & =[x, z]+[y, z], \\
{[\lambda x, y] } & =\lambda[x, y], \\
{[x, x] } & >0 \text { for } x \neq 0, \quad \text { for } x, y, z \text { in } X \text { and } \lambda \in C . \\
\|[x, y]\|^{2} & \leqslant[x, x][y, y]
\end{aligned}
$$

By the Hahn-Banach theorem, there is a semi-inner-product with $\|x\|$ $=[x, x]^{1 / 2}$.

Corollary 3.3. Suppose $F \in \bar{U}_{H, C}$, and let

$$
h(t)=\lim _{s \rightarrow 0^{+}} s^{-1}\left(\left\|I+s D F_{x(t)}\right\|-1\right),
$$

where $x(t)=t F(x(t))$ for $0<t<1$. If $h(t) \leqslant 1$ and $(1-t h(t))^{-1}$ is integrable on $(1-\delta, 1)$ for some sufficiently small $\delta$, then $F$ has a fixed point in $\bar{U}$.

Proof. The condition $h(t) \leqslant 1$ is shown in Lumer and Phillips [11] to imply that $\operatorname{Re}\left[D F_{x(t)} y, y\right] \leqslant h(t)\|y\|^{2}$. Hence

$$
\begin{aligned}
\left\|\left(I-t D F_{x(t)}\right) y\right\|\|y\| & \geqslant \operatorname{Re}\left[\left(I-t D F_{x(t)}\right) y, y\right]=\operatorname{Re}[y, y]-t \operatorname{Re}\left[D F_{x(t)} y, y\right] \\
& \geqslant\|y\|^{2}-t h(t)\|y\|^{2} .
\end{aligned}
$$

Therefore $I-t D F_{x(t)}$ is invertible and $\left\|\left(I-t D F_{x(t)}\right)^{-1}\right\| \leqslant(1-t h(t))^{-1}$. An application of Theorem 3.2 shows that the length of the path of $x(t)$ $=t F(x(t))$ is finite, so $F$ has a fixed point.

The condition in the last corollary is related to pseudo-contractive maps introduced by Browder [1] and to accretive operators, but is slightly stronger. To see the connection we suppose for a moment that $A=D F_{x(t)}$ is linear and $X$ is a Hilbert space. In our last corollary we have the condition that $\operatorname{Re}(A y, y) \leqslant h(t)\|y\|^{2}$. If $\operatorname{Re}(A y, y) \leqslant\|y\|^{2}$ then $\operatorname{Re}((I-A) y, y) \geqslant 0$ or $I$ $-A$ is accretive. $I-A$ is accretive if and only if $A$ is pseudo-contractive. So for linear operators, pseudo-contractive implies the numerical range of $A$ is in a half plane with real part less than or equal to one. In the corollary we needed 
to restrict the numerical range in a slight shift to the left. If we impose the condition $\operatorname{Re}(A x, x) \leqslant k\|x\|^{2}$ for $k<1$, then $k^{-1} A$ is pseudo-contractive. We use these comments to generalize to the nonlinear case. Following Browder [1] we say a map $G: D \rightarrow X$, where $D$ is the domain of $G$ and $X$ is a Banach space, is pseudo-contractive if for all $u, v$ in $D$ and $r>0$,

$$
\|u-v\| \leqslant\|(1+r)(u-v)-r(G(u)-G(v))\| .
$$

Browder and Kato have shown that in the nonlinear setting this is equivalent to $I-G$ accretive. We will essentially require that $I-G$ be strictly accretive, or, equivalently, following [3], say a map $G: D \rightarrow X$ is strictly pseudocontractive if for $0<k<1$ and $0<\lambda<1 / k$ we have $\|(1-\lambda G) x-$ $(I-\lambda G) y\|\geqslant(1-\lambda k)\| x-y \|$. Note when $k=1$ and $\lambda=r(1+r)^{-1}$ we have a pseudo-contractive map. Browder [1] and Kirk [10] have used the pseudocontractive maps and additional conditions in uniformly convex spaces to prove fixed point theorems. The relationship of strictly pseudo-contractive maps to monotone maps, iterative methods for the construction of their fixed points in Hilbert space, and historical remarks may be found in [3]. Extending the result of Kirk [10] we have the following theorem.

Theorem 3.4. Suppose $X$ is a Banach space, $F: U \rightarrow U$ is strictly pseudocontractive and the range of $(I-\lambda F) U$ contains an open ball of radius $1-\lambda$ about the origin for some $\lambda, 0<\lambda<1$. Then $F$ has a fixed point in $U$.

Proof. Let $T_{\lambda}=I-\lambda F$. Then strictly pseudo-contractive implies that $\left\|T_{\lambda} x-T_{\lambda} y\right\| \leqslant(1-k \lambda)\|x-y\|$ or that $T_{\lambda}^{-1}\left[B_{1-\lambda}\right] \subset U$ where $B_{1-\lambda}$ is the open ball of radius $1-\lambda$ and center 0 . Hence $(1-\lambda) T_{\lambda}^{-1}: B_{1-\lambda} \rightarrow B_{1-\lambda}$ and since $1-\lambda<1-k \lambda,(1-\lambda) T_{\lambda}^{-1}$ is a strict contraction. Let $x \in B_{1-\lambda}$ be the fixed point of $(1-\lambda) T_{\lambda}^{-1}$. Suppose $z=x(1-\lambda)^{-1}$, then $T_{\lambda}(z)=x$ or $z-\lambda F(z)=x=(1-\lambda) z$ or $F(z)=z$.

Corollary 3.5. Suppose $X$ is a Banach space and $F: U \rightarrow U$ is strictly pseudo-contractive and holomorphic; then $F$ has a fixed point in $U$.

Proof. We need to show $R(I-\lambda F)[U] \supset B_{1-\lambda}$. Let $F_{\lambda}=\lambda F$ for $0<\lambda$ $<1$, and let $y \in B_{1-\lambda}$ so that $\|y\| \leqslant \varepsilon(1-\lambda)$ for some $\varepsilon<1$. Let $G_{\lambda}$ $=\lambda F+y$. Then $G_{\lambda}$ is holomorphic and maps $U$ strictly inside $U$. Hence, $G_{\lambda} x=x=\lambda F(x)+y$ or $(I-\lambda F) x=y$. This shows that $R(I-\lambda F)[U]$ $\supset B_{1-\lambda}$.

REMARKS. Following Kirk [10] we see that the conditions strictly pseudocontractive and Lipschitzian on $F$ will yield the above corollary. Also if the space is uniformly convex and $F$ is pseudo-contractive on $\bar{U}$ and holomorphic in some open ball containing $\bar{U}$, and $F: \bar{U} \rightarrow \bar{U}$ then $F$ has a fixed point in $\bar{U}$. Finally we remark that some of the above results remain true if $U$ is replaced by a bounded star shaped domain.

4. Linear fractional transformations. We will show that some linear fractional maps have fixed points. Krein [9] showed that there is an interesting relation 
between the fixed points of linear fractional transformations and invariant subspace problems in a Hilbert space with indefinite metric. Phillips [14] generalized the results of C. L. Siegel [16] and was able to characterize the linear fractional maps of Krein as biholomorphic maps on the unit ball of $B(H, H)$, the bounded linear operators on a Hilbert space $H$, which are in the identity component.

Helton [7] exploited the relation between invariant subspaces and fixed points established by Krein and Phillips and characterized those maps of Phillips having fixed points in the interior of the unit ball. L. Harris has considered biholomorphic maps on the unit ball of $B(H, H)$ and was able to characterize such maps by $h(Z)=L \circ T_{S}(Z)$ where $L$ is a linear isometry and

$$
T_{S}(Z)=\left(I-S S^{*}\right)^{-1 / 2}(S-Z)\left(I-S^{*} Z\right)^{-1}\left(I-S^{*} S\right)^{1 / 2}
$$

and $\|S\|<1$. For a discussion of this result and its relation to the result of Phillips see Harris [5].

We are able to show that $T_{S}(Z)$ has a fixed point in the interior of the unit ball and in fact are able to explicitly compute the fixed point. Note that this is equivalent to solving a rather complicated "quadratic" equation in operators.

THEOREM 4.1. The map $T_{S}(Z)=A^{-1 / 2}(S-Z)\left(I-S^{*} Z\right)^{-1} B^{1 / 2}$ (where $A=I-S S^{*}, B=I-S^{*} S$, and $\left.\|S\|<1\right)$ is a biholomorphic map of the unit ball of $B(H, H)$ onto itself and the point $K=S\left(I+B^{1 / 2}\right)^{-1}$ is a fixed point of $T_{S}$ inside the unit ball.

Proof. First note that $A$ and $B$ are positive operators and $0<A \leqslant I, 0$ $<B \leqslant I$, so that $B$ has a positive square root and $\left(I+B^{1 / 2}\right)^{-1}$ has norm less than one so that $\|K\|<1$.

We will show that $(S-K)\left(I-S^{*} K\right)^{-1}=A^{1 / 2} K B^{-1 / 2}$. Now

$$
\begin{aligned}
(S-K)\left(I-S^{*} K\right)^{-1} & =\left[S-S\left(I+B^{1 / 2}\right)^{-1}\right]\left[I-S^{*} S\left(I+B^{1 / 2}\right)^{-1}\right]^{-1} \\
& =S\left[I-\left(I+B^{1 / 2}\right)^{-1}\right]\left[I-\left(I-B^{1 / 2}\right)\right]^{-1} \\
& =S\left(I-\left(I+B^{1 / 2}\right)^{-1}\right) B^{-1 / 2} .
\end{aligned}
$$

Note that for any positive integer $n, S\left(S^{*} S\right)^{n}=\left(S S^{*}\right)^{n} S$ and hence for $\|S\|<1$, if $\sum a_{n} z^{n}$ converges in the unit circle we have $\sum a_{n} S\left(S^{*} S\right)^{n}$ $=\sum a_{n}\left(S S^{*}\right)^{n} S$. Or for $f$ analytic on $|z|<1, S f\left(S^{*} S\right)=f\left(S S^{*}\right) S$ and, in particular, $S\left(I-S^{*} S\right)^{1 / 2}=\left(I-S S^{*}\right)^{1 / 2} S$. Hence

$$
\begin{gathered}
S B^{1 / 2}=A^{1 / 2} S=S\left(I+B^{1 / 2}-I\right) \quad \text { or } \\
A^{1 / 2} S\left(I+B^{1 / 2}\right)^{-1}=S\left[I-\left(I+B^{1 / 2}\right)^{-1}\right] .
\end{gathered}
$$

Multiply by $B^{-1 / 2}$ and use the relation obtained above to see that $(S-K)$ $\cdot\left(I-S^{*} K\right)^{-1}=A^{1 / 2} K B^{-1 / 2}$, so that $K$ is a fixed point. 
In closing we mention without proof the following partial result obtained in joint work with L. Harris in Hilbert space. Suppose $F: \bar{U} \rightarrow \bar{U}$ is continuous on $\bar{U}$ and holomorphic in $U$. In case the approximate fixed points $x(t)$ of the map $t F, 0 \leqslant t<1$, satisfy either of the following two conditions

(a) $\|x(t)\| \leqslant r<1$,

(b) $\|x(t)\| \geqslant t^{n}$ for some $n \geqslant 1$ as $t \rightarrow 1$, then $F$ has a fixed point.

The authors thank Frank Massey and L. Harris for helpful discussions. The first author wishes to thank Ralph Phillips for bringing to his attention the problem of Krein while the first author was on a sabbatical leave at Stanford which initiated interest in the problems of this paper.

Appendix. These results are due to $\mathrm{L}$. Harris and generalize the results of Earle and Hamilton [4]. In a related way, Marsden [12] has used the semigroup structure of a flow to define a metric in which the flow is globally well behaved.

THEOREM 1. Let $\mathcal{D}$ be an open connected subset of a normed linear space $X$ and let $\delta$ be a semigroup of continuously differentiable functions $h: \mathscr{D} \rightarrow \mathscr{D}$, where the semigroup operation is composition of functions. Suppose that the map $x$ $\rightarrow \sup _{h \in \delta}\|D h(x)\|$ is locally bounded. Then there is a metric $\rho$ on $\mathcal{D}$ with $\rho(h(x), h(y)) \leqslant \rho(x, y)$ for all $h \in \mathcal{S}$ and all $x, y \in \mathscr{D}$. Moreover $\rho(x, y)$ $\geqslant\|y-x\|$ for all $x, y \in \mathscr{D}$, and given a point in $\mathscr{D}$ there is a neighborhood $\theta$ of the point and a real number $M$ such that $\rho(x, y) \leqslant M\|x-y\|$ for all $x, y \in \mathcal{O}$.

Conversely, it is easy to show that if $\rho$ is a metric on $\mathscr{D}$ which satisfies the conclusions of the above theorem, then the map $x \rightarrow \sup _{h \in \mathcal{S}}\|D h(x)\|$ is locally bounded. If $\mathscr{D}$ is bounded, the semigroup $\delta$ of all holomorphic functions $h: \mathscr{D} \rightarrow \mathscr{D}$ satisfies the above hypotheses; for if $x \in \mathscr{D}$, there exist $r, R$ $>0$ with $B_{2 r}(x) \subseteq \mathcal{D} \subseteq B_{R}(0)$. Hence given $y \in B_{r}(x)$, we may apply the Cauchy estimates on $B_{r}(y)$ to any function $h \in \delta$ to obtain $\|D h(y)\| \leqslant R / r$. Thus $x \rightarrow \sup _{h \in \delta}\|D h(x)\|$ is locally bounded. Clearly the topology generated by the metric $\rho$ is equivalent to the norm topology.

Proof. We may suppose that the identity map $I$ is in $\mathcal{S}$. Define

$$
\alpha(x, y)=\sup _{h \in \mathcal{S}}\|D h(x) y\|
$$

for $x \in \mathscr{D}$ and $y \in X$. Let $\gamma$ be a continuous curve in $\mathscr{D}$ whose derivative is continuous except possibly at finitely many points. (We call such a curve admissible.) Since the range of $\gamma$ is compact, there is an $M$ with $\|D h(\gamma(t))\|$ $\leqslant M$ for all $h \in \mathcal{S}$ and $0 \leqslant t \leqslant 1$, so $\alpha\left(\gamma(t), \gamma^{\prime}(t)\right) \leqslant M\left|\gamma^{\prime}(t)\right|$ for $0 \leqslant t \leqslant 1$. Define $L(\gamma)=\int_{0}^{1} \alpha\left(\gamma(t), \gamma^{\prime}(t)\right) d t$ and take $\rho(x, y)=\inf \{L(\gamma): \gamma$ admissible, $\gamma(0)=x, \gamma(1)=y\}$. It is easy to show that $\rho$ is a metric. Suppose $h \in \delta$ and let $u \in \mathscr{D}$ and $v \in X$. Then by the chain rule, for any $g \in \mathcal{S}$,

$$
\|D g(h(u)) D h(u) v\|=\|D(g \circ h)(u) v\| \leqslant \alpha(u, v)
$$

since $\delta$ is a semigroup. Hence 


$$
\alpha(h(u), D h(u) v) \leqslant \alpha(u, v) .
$$

Now let $\gamma$ be an admissible curve with $\gamma(0)=x$ and $\gamma(1)=y$. Then $x \circ \gamma$ is also an admissible curve and $(h \circ \gamma)(0)=h(x),(h \circ \gamma)(1)=h(y)$. By the chàin rule,

$$
\begin{aligned}
L(h \circ \gamma) & =\int_{0}^{1} \alpha\left((h \circ \gamma)(t),(h \circ \gamma)^{\prime}(t)\right) d t=\int_{0}^{1} \alpha\left(h(\gamma(t)), D h(\gamma(t)) \gamma^{\prime}(t)\right) d t \\
& \leqslant \int_{0}^{1} \alpha\left(\gamma(t), \gamma^{\prime}(t)\right) d t=L(\gamma),
\end{aligned}
$$

where the last inequality follows from $(*)$. Hence $\rho(h(x), h(y)) \leqslant \rho(x, y)$.

To see that $\rho(x, y) \geqslant\|y-x\|$, let $\gamma$ be an admissible curve in $\mathscr{D}$ with $\gamma(0)=x$ and $\gamma(1)=y$. Then

$$
\begin{aligned}
L(\gamma) & =\int_{0}^{1} \alpha\left(\gamma(t), \gamma^{\prime}(t)\right) d t \geqslant \int_{0}^{1}\left\|\gamma^{\prime}(t)\right\| d t \geqslant\left\|\int_{0}^{1} \gamma^{\prime}(t) d t\right\| \\
& =\|\gamma(1)-\gamma(0)\|=\|y-x\|,
\end{aligned}
$$

so $\rho(x, y) \geqslant\|y-x\|$. (It is obvious that $\alpha(u, v) \geqslant\|v\|$ for $u \in \mathscr{D}, v$ $\in X$ since $I \in \delta$.)

Finally, let $p$ be a point of $\mathscr{D}$. Then there is a ball $\theta$ with center $p$ and a constant $M$ with $\sup _{h \in \delta}\|D h(x)\| \leqslant M$ for all $x \in \mathcal{O}$. Let $x, y \in \mathcal{O}$ and put $\gamma(t)=x+t(y-x)$. Then

$$
\alpha\left(\gamma(t), \gamma^{\prime}(t)\right)=\sup _{h \in \delta}\left\|D h(\gamma(t)) \gamma^{\prime}(t)\right\| \leqslant M\|y-x\|
$$

so

$$
\rho(x, y) \leqslant L(\gamma)=\int_{0}^{1} \alpha\left(\gamma(t), \gamma^{\prime}(t)\right) d t \leqslant M\|y-x\|
$$

for all $x, y \in \mathcal{C}$.

An $h \in \delta$ is said to be a $(C)$-element of $\delta$ if there is a $t>0$ such that the function $x \rightarrow h(x)+t[h(x)-y]$ belongs to $s$ whenever $y \in \mathscr{D}$.

THEOREM 2. Suppose $\delta$ satisfies the hypotheses of Theorem 1 above. If $h$ is a (C)-element of $\mathcal{S}$, then

$$
\alpha(h(x), D h(x) v) \leqslant k \alpha(x, y) \text { and } \rho(h(x), h(y)) \leqslant k \rho(x, y)
$$

for all $x, y \in \mathscr{D}$ and all $v \in X$, where $k=(1+t)^{-1}$. Moreover, if $X$ is complete, then $h$ has a fixed point in $\mathscr{D}$.

Proof. Let $x \in \mathscr{D}$ and define $g(y)=h(y)+t[h(y)-h(x)]$ for $y \in \mathscr{D}$. By hypothesis, $g \in \mathfrak{S}$, so by $(*)$,

$$
\alpha(g(x), D g(x) v) \leqslant \alpha(x, v), \quad \text { i.e. } \alpha(h(x),(1+t) D h(x) v) \leqslant \alpha(x, v)
$$

for all $v \in X$, as desired. It is easy to modify the corresponding argument in 
the proof of Theorem 1 to show that the last inequality implies that

$$
\rho(h(x), h(y)) \leqslant(1+t)^{-1} \rho(x, y) .
$$

The last part of the theorem follows immediately from the contraction mapping theorem.

For example, suppose $\mathscr{D}$ is bounded and that $h: \mathscr{D} \rightarrow \mathscr{D}$ is a holomorphic function which maps $\mathscr{D}$ strictly inside itself (i.e., there is an $\varepsilon>0$ such that $\|h(x)-y\| \geqslant \varepsilon$ whenever $x \in \mathscr{D}$ and $y \notin \mathscr{D})$. Then it is easy to see that $h$ is a $(C)$-element of the semigroup $\delta$ of all holomorphic functions $f: \mathscr{D} \rightarrow \mathscr{D}$, and consequently the conclusions of Theorem 2 hold. (This is the main result of [4].)

REMARK. If $\mathscr{D}$ is the open unit ball of a normed linear space and if $\delta$ is a semigroup of holomorphic functions mapping $\mathscr{D}$ into $\mathscr{D}$, then

$$
\|x\| \leqslant \rho(0, x) \leqslant \tanh ^{-1}\|x\|
$$

for all $x \in \mathcal{D}$.

Proof. Given $x \in \mathcal{D}$ with $x \neq 0$ and $h \in \mathcal{S}$, by the Hahn-Banach theorem there is an $l \in X^{*}$ with $l(D h(x) x)=\|D h(x) x\|$ and $\|l\|=1$. Define $f(z)=l(h(z x /\|x\|))$. Clearly $f$ is an analytic function in the disc $|z|<1$ in the classical sense and satisfies $|f(z)| \leqslant 1$ there. Then, as is well known, $\left|f^{\prime}(\|x\|)\right|$ $\leqslant\left(1-\|x\|^{2}\right)^{-1}$, so

$$
\|D h(x) x\| \leqslant\|x\| /\left(1-\|x\|^{2}\right) .
$$

Thus $\alpha(x, x) \leqslant\|x\|\left(1-\|x\|^{2}\right)^{-1}$ for all $x \in \mathscr{D}$. Therefore given $x \in \mathscr{D}$ and taking $\gamma(t)=t x$ for $0 \leqslant t \leqslant 1$, we have

$$
\begin{aligned}
\rho(0, x) & \leqslant L(\gamma)=\int_{0}^{1} \alpha(t x, x) d t=\int_{0}^{1} \frac{1}{t} \alpha(t x, t x) d t \\
& \leqslant \int_{0}^{1}\|x\|\left(1-t^{2}\|x\|^{2}\right)^{-1} d t=\frac{1}{2} \log \frac{1+\|x\|}{1-\|x\|}=\tanh ^{-1}\|x\| .
\end{aligned}
$$

Note that the inequality $\log (1+t) \leqslant t$ implies that

$$
\tanh ^{-1} r \leqslant r(1-r)^{-1} \text { for } 0 \leqslant r<1 .
$$

\section{REFERENCES}

1. F. E. Browder, Nonlinear mappings of nonexpansive and accretive type in Banach spaces, Bull. Amer. Math. Soc. 73 (1967), 875-882. MR 38 \#581.

2. F. E. Browder and W. V. Petryshyn, The solution by iteration of nonlinear functional equations in Banach spaces, Bull. Amer. Math. Soc. 72 (1966), 571-575. MR 32 \#8155b.

3. - Construction of fixed points of nonlinear mappings in Hilbert space, J. Math. Anal. Appl. 20 (1967), 197-228. MR 36 \#477.

4. C. J. Earle and R. S. Hamilton, A fixed point theorem for holomorphic mappings, Proc. Sympos. Pure Math., vol. 16, Amer. Math. Soc., Providence, R. I., 1970, pp. 61-65. MR 42 \# 918.

5. L. A. Harris, Bounded symmetric homogeneous domains in infinite dimensional spaces, Lecture 
Notes in Math., vol. 364, Springer-Verlag, Berlin and New York, 1974, pp. 13-39.

6. T. L. Hayden and T. J. Suffridge, Biholomorphic maps in Hilbert space have a fixed point, Pacific J. Math. 38 (1971), 419-422. MR 46 \#4288.

7. J. W. Helton, Operators unitary in an indefinite metric and linear fractional transformations, Acta Sci. Math. 32 (1971), 261-266.

8. E. Hille and R. S. Phillips, Functional analysis and semi-groups, rev. ed., Amer. Math. Soc. Colloq. Publ. vol. 31, Amer. Math. Soc., Providence, R. I., 1957. MR 19, 664.

9. M. G. Krein, $A$ new application of the fixed-point principle in the theory of operators on a space with indefinite metric, Dokl. Akad. Nauk SSSR 154 (1964), 1023-1026 = Soviet Math. Dokl. 5 (1964), 224-228. MR 29 \#6314.

10. W. A. Kirk, Remarks on pseudo-contractive mappings, Proc. Amer. Math. Soc. 25 (1970), 820-823. MR 41 \#9074.

11. G. Lumer and R. S. Phillips, Dissipative operators in a Banach space, Pacific J. Math. 11 (1961), 679-698. MR 24 \#A2248.

12. J. Marsden, On product formulas for nonlinear semigroups, J. Functional Analysis 13 (1973), 51-72. MR 50 \#156.

13. W. V. Petryshyn, Construction of fixed points of demicompact mappings in Hilbert space, J. Math. Anal. Appl. 14 (1966), 276-284. MR 33 \#3147.

14. R. S. Phillips, On symplectic mappings of contraction operators, Studia Math. 31 (1968), 15-27. MR 38 \# 5048.

15. R. Ryan, Boundary values of analytic vector valued functions, Nederl. Akad. Wetensch Proc. Ser. A 65 = Indag. Math. 24 (1962), 558-572. MR 26 \#2621.

16. C. L. Siegel, Symplectic geometry, Amer. J. Math. 65 (1943), 1-86. MR 4, 242.

Department of Mathematics, University of Kentucky, Lexington, Kentucky 40506 